\title{
Access to hyperacute stroke services across Canadian provinces: a geospatial analysis
}

\author{
Prasanna Venkatesan Eswaradass MD, Richard H. Swartz MD PhD, Jamey Rosen MSc PGeo, \\ Michael D. Hill MD MSc, M. Patrice Lindsay BScN PhD
}

\section{Abstract}

Background: Canada's vast geography creates challenges for ensuring prompt transport to hospital of patients who have had a stroke. We sought to determine the proportion of people across various Canadian provinces for whom hyperacute stroke services are accessible within evidence-based time targets.

Methods: We calculated, for the 8 provinces with available data, drive-time polygons on a map of Canada that delineated the area around stroke centres and emergency medical services (EMS) base centres to which one can drive in 3.5-6 hours. We calculated the proportional area of each forward sortation area (first 3 digits of the postal code) contained within a drive-time polygon. We applied this ratio to the 2011 Canadian census population of the forward sortation area to estimate the population that can reach a stroke centre in a designated time.

Results: A total of $47.1 \%-96.4 \%$ of Canadians live within a 4.5 -hour drive to a stroke centre via road EMS, and $53.3 \%-96.8 \%$ live within a 6-hour drive. Assuming a total travel time of 5 hours by EMS from base centre to patient and patient to hospital, $84.7 \%-99.8 \%$ of the population has access to a current or proposed endovascular thrombectomy site.

Interpretation: Most Canadians live within 6 hours' road access to a stroke centre. Geospatial mapping could be used to inform decisions for additional sites and identify gaps in service accessibility. Coordinated systems of care and ambulance bypass agreements must continue to evolve to ensure maximal access to time-sensitive emergency stroke services.

troke is an acute neurologic emergency with major public health impact. ${ }^{1}$ Hyperacute treatment for stroke is evolving rapidly, with implications for service delivery and for equitable access to evolving standards of care. About $85 \%$ of strokes in North America are ischemic. The primary treatment of acute ischemic stroke is intravenous administration of alteplase, ${ }^{2}$ but outcome is highly associated with speed of access, measured by onset-to-treatment time., As per Canadian best-practice recommendations, intravenously administered alteplase is offered for disabling stroke within 4.5 hours of stroke onset, and endovascular thrombectomy is offered for strokes due to large-vessel occlusion within 6 hours. $^{5}$ Both primary and comprehensive stroke centres offer medical treatment with alteplase. However, endovascular thrombectomy is a newer therapy that is available in a limited number of comprehensive stroke centres with the necessary equipment and professional expertise to perform the procedure and care for the patient afterward.

Most patients who have a stroke do not receive treatment because they present too late or have medical contraindications to treatment. Stroke centres and dedicated stroke teams can continually improve the quality of treatment offered to patients who arrive within evidence-based time windows to warrant treatment. Implementation of a comprehensive system of stroke care has increased the role of emergency medical services (EMS) for transport of patients with stroke.

Early identification of stroke and protocols to enable rapid transport via EMS have resulted in reduced delay in treatment, reduced interfacility transfer delays and increased rates of thrombolysis administration for acute ischemic stroke. ${ }^{6-8}$ The recent establishment of endovascular therapy for patients with ischemic stroke places even greater demands on systems of care, with fewer centres able to support neurointervention around-the-clock 7 days a week. Every minute of delay in reperfusion results in a loss of 1.9 million neurons. ${ }^{9}$ With endovascular therapy, the odds of benefit reduce with every hour of delay from stroke onset to arterial puncture. ${ }^{10}$ Given the need for rapid treatment, geographically dispersed popu-

Competing interests: See the end of the article.

This article has been peer reviewed.

Correspondence to: Patrice Lindsay, patrice.lindsay@ heartandstroke.ca CMAJ Open 2017. DOI:10.9778/cmajo.20160166 
lations may not be able to benefit from either alteplase or endovascular thrombectomy.

Canada's vast geography creates challenges for ensuring prompt transport to hospital. To inform ongoing efforts to plan and improve the health care system with the goal of optimizing delivery of stroke services, we sought to determine the proportion of people across several provinces for whom hyperacute stroke services are currently accessible via EMS.

\section{Methods}

We estimated the population with access to stroke centres in sufficient time for treatment, by province, using the 2011 Canadian population census. We compared the area within which residents could be reached by an ambulance (travelling by road) and then driven to a stroke hospital to the population in those regions (based on forward sortation area [first 3 digits of postal code]) to determine the proportion of residents who could reach a hospital 3.5-6 hours after contacting EMS or by driving themselves to hospital (Figure 1). Postal codes in Canada are assigned based on a combination of geography, population and city planning. Generally speaking, more densely populated areas have smaller postal code geographic areas than do more sparsely populated areas.

We used geospatial analyses with geographic information systems technology ${ }^{11-13}$ to generate drive-time polygons. A drive-time polygon represents a shape on a map that contains all points a driver can theoretically reach from a starting point by personal vehicle within the time allotted. We geocoded the addresses of hospitals with comprehensive and advanced stroke centres and of EMS centres (defined as locations of paramedic base stations) to assign geographic coordinates (latitude and longitude). These addresses were available for 8 provinces (EMS centre locations for Nova Scotia and Quebec were not available); therefore, the analysis was limited to those regions. Once geocoding was complete, we calculated drive-time polygons for each hospital and EMS centre using the ArcGIS World Route service algorithm. This algorithm delineates the spatial extent to which one can drive from a starting point within a given duration, assuming average driving times per road segment. The algorithm assumes average civilian driving times, but drivers of emergency vehicles generally drive faster than civilians, which results in larger drive-time polygons. The assumption results in an underestimation of the population with sufficient access to stroke centres. The algorithm accounts for bridges, water transport, historical traffic patterns and other permanent impediments to driving but not transient features such as construction and road traffic.

We calculated drive-time polygons with the simplified assumption that at least half of the total journey to the hospital would be spent by an ambulance travelling to the patient's location and half by the ambulance's travelling from the patient's location to the stroke centre. Drive-time polygons were thus calculated at 1.75, 2.25, 2.5 and 3 hours (i.e., half of $3.5,4.5,5$ and 6 hours' total driving time) around each stroke centre and EMS centre. The actual duration (in addition to driving times) would be increased by the on-site time incurred by EMS personnel (median 16-24 min across provinces). ${ }^{1}$ Thus, the driving time of 3.5 hours is potentially closer to 3 hours and 45 minutes when on-site time is included. Drivetime polygons were then spatially intersected with forward sortation areas to identify the regions within which residents lived with access to both a stroke centre and an EMS drivetime polygon (the "serviced region"). This method assumed that road ambulance was the only method by which residents could travel to a stroke centre.

We used forward sortation areas with associated 2011 populations to estimate the population inside and outside the

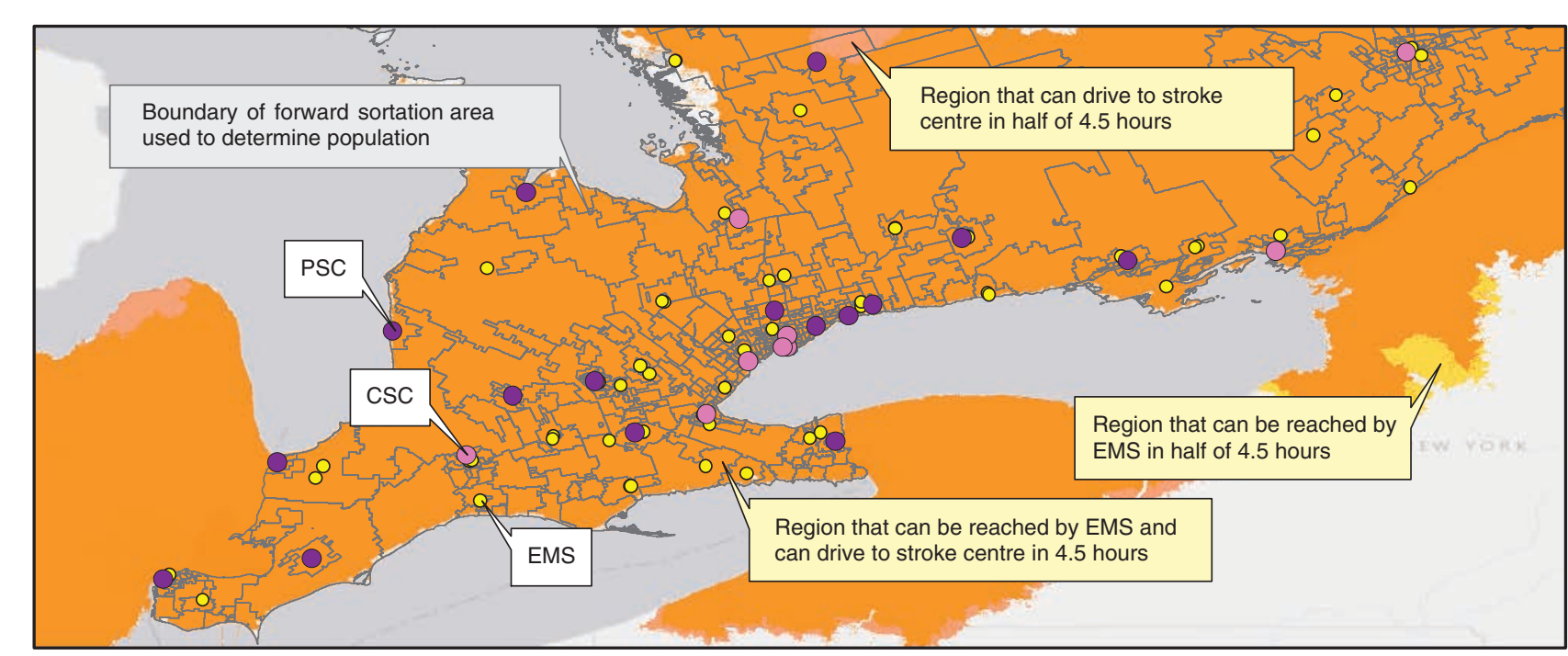

Service Layer Credits: Esri, HERE, DeLorme, MapmyIndia, @ OpenStreetMap contributors, and the GIS user community

Figure 1: Map of southwestern Ontario, showing multiple comprehensive stroke centres (CSCs) (pink), primary stroke centres (PSCs)(purple) and colour-coded drive-time polygons. EMS = emergency medical services. 
serviced region. The ratio of the serviced area to the forward sortation area multiplied by the population within the associated forward sortation area represents the portion of residents with access to a stroke centre within the treatment time window.

We summed the populations within the serviced regions for each province to calculate provincial totals. This method assumed that residents had a stroke at home (or at a location within their home forward sortation area).

A major Canadian clinical trial on endovascular care (ESCAPE [Endovascular Treatment for Small Core and Anterior Circulation Proximal Occlusion with Emphasis on Minimizing CT to Recanalization Times]) included 11 sites, and an additional 10 sites have implemented this treatment or are in the planning stages. ${ }^{14} \mathrm{We}$ conducted a subanalysis to determine access to acute endovascular thrombectomy for large-vessel ischemic stroke across the country.

\section{Results}

Available data allowed us to include 456 EMS centres and 637 hospitals and to calculate the drive-time polygons on a map of the 8 provinces studied. Alberta (229 EMS centres and 96 hospitals, of which 20 were stroke centres) and Ontario (82 EMS main base centres and additional local centres, and 165 hospitals, of which 38 were stroke centres) had the largest numbers of EMS centres and hospitals in the country. Two stroke centres in Alberta were ESCAPE endovascular thrombectomy sites, and 5 stroke centres in Ontario were ESCAPE sites, with 4 additional sites planned. Prince Edward Island had 6 EMS centres and 4 hospitals. Overall, 69.0\% of patients admitted to hospital with stroke in Canada were transported to the emergency department by EMS (Table 1).
An estimated $78.9 \%$ of the population of the 8 provinces studied had predicted access to hyperacute stroke services via EMS or self-driving within 4.5 hours. This represents the maximum proportion of the population who could benefit from time-sensitive therapies. As an example, $89.3 \%, 91.6 \%$ and $93.0 \%$ of the population of Alberta (3 645081 in 2011) lived within a 3.5-, 4.5- and 6-hour drive, respectively, from a stroke centre via EMS (Figure 2); the corresponding proportions for

\begin{tabular}{|c|c|}
\hline Province & No. $(\%)$ of patients \\
\hline British Columbia & $4704 / 7002(67.2)$ \\
\hline Alberta & $3219 / 4306(74.8)$ \\
\hline Saskatchewan & 1072/1679 (63.8) \\
\hline Manitoba & $1098 / 1689(65.0)$ \\
\hline Ontario & 12 464/17 939 (69.5) \\
\hline New Brunswick & $832 / 1352(61.5)$ \\
\hline Nova Scotia & 1069/1465 (73.0) \\
\hline Prince Edward Island & $174 / 272(64.0)$ \\
\hline Newfoundland and Labrador & $610 / 881(69.2)$ \\
\hline Canada & 25 242/36 585 (69.0) \\
\hline \multicolumn{2}{|c|}{$\begin{array}{l}\text { *Some of these data are based on information provided by the Canadian } \\
\text { Institute for Health Information (CIHI). However, the analyses, conclusions, } \\
\text { opinions and statements expressed herein are those of the authors and not } \\
\text { those of the ClHI. } \\
\text { tData on emergency medical services transport were not collected or reported } \\
\text { in Quebec at the time of analysis. }\end{array}$} \\
\hline
\end{tabular}

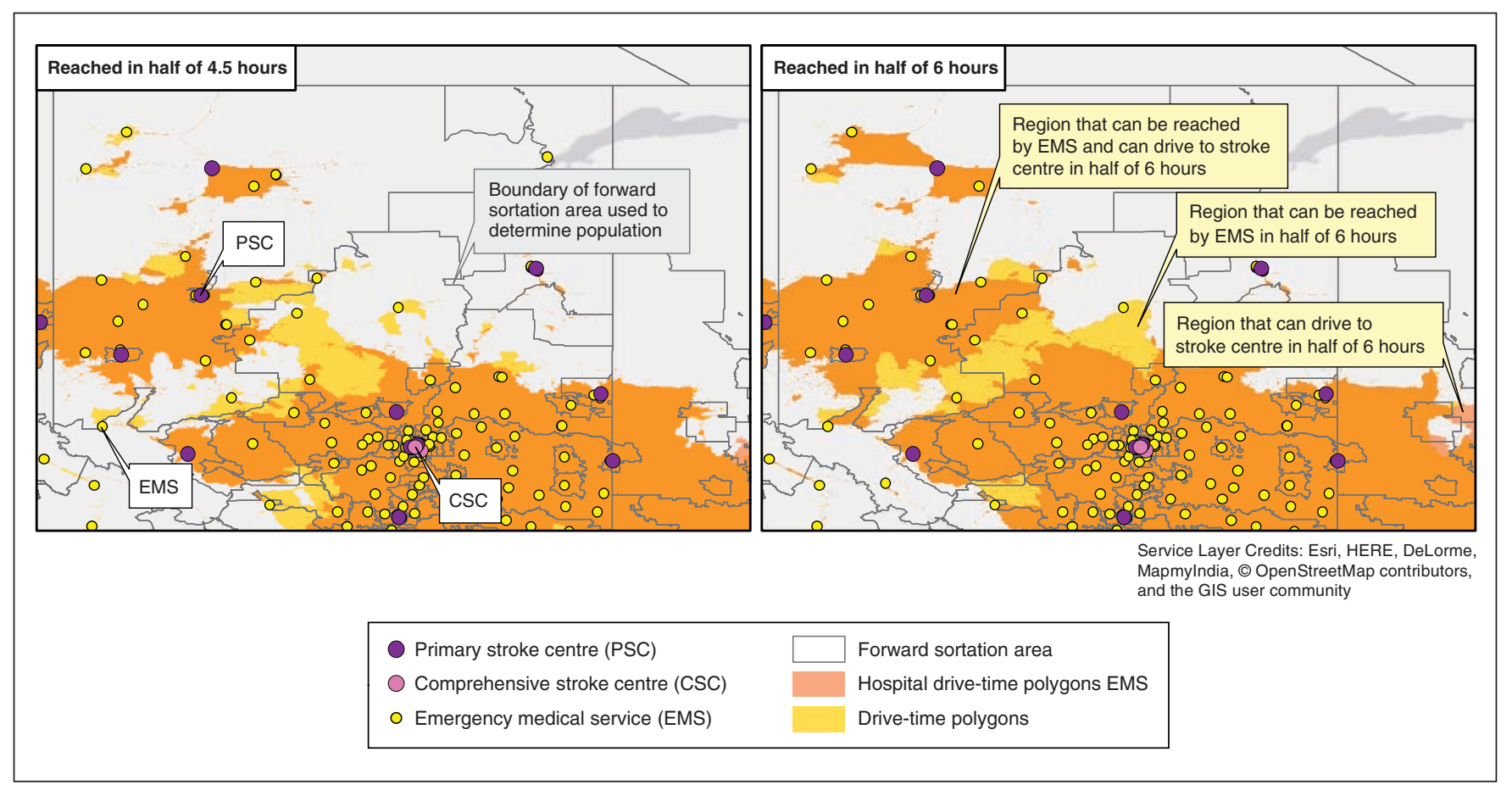

Figure 2: Selected map of northern Alberta with Edmonton noted as the comprehensive stroke centre, showing colour-coded drive-time polygons. 
self-driving were $94.3 \%, 98.4 \%$ and $98.7 \%$. The mean proportion of the Alberta population living within serviced regions was $92.4 \%$. For Ontario, $95.6 \%, 96.4 \%$ and $97.3 \%$ of the population (12 851791 in 2011) lived within 3.5, 4.5 and 6 hours, respectively, from a stroke centre via EMS; the corresponding proportions for self-driving were $97.7 \%, 99.5 \%$ and $99.6 \%$ (Table 2). Other provinces showed similar results.

\begin{tabular}{|c|c|c|}
\hline Province* & $\begin{array}{l}2011 \text { census } \\
\text { population }\end{array}$ & No. (\%) with access $†$ \\
\hline British Columbia & 4400052 & $3356102(76.3)$ \\
\hline Alberta & 3645081 & 3340445 (91.6) \\
\hline Saskatchewan & 1033489 & $534826(51.7)$ \\
\hline Manitoba & 1208268 & $1057871(87.6)$ \\
\hline Ontario & 12851791 & 12396339 (96.4) \\
\hline New Brunswick & 751161 & $676261(90.0)$ \\
\hline $\begin{array}{l}\text { Prince Edward } \\
\text { Island }\end{array}$ & 140204 & $126608(90.3)$ \\
\hline $\begin{array}{l}\text { Newfoundland and } \\
\text { Labrador }\end{array}$ & 514536 & 242419 (47.1) \\
\hline \multicolumn{3}{|c|}{$\begin{array}{l}\text { *Emergency medical services (EMS) base locations not available for Nova } \\
\text { Scotia or Quebec. } \\
\text { tAssumption that driving time of } 4.5 \text { hours is divided, with } 50 \% \text { of time from } \\
\text { EMS starting location to patient and the other } 50 \% \text { from patient location to } \\
\text { closest stroke centre. }\end{array}$} \\
\hline
\end{tabular}

Only $41.3 \%$ and $44.2 \%$ of the populations of Saskatchewan and of Newfoundland and Labrador, respectively, lived within 3.5 hours from a stroke centre via EMS; the corresponding proportions increased to $51.7 \%$ and $47.1 \%$ for 4.5 hours and $84.0 \%$ and $53.3 \%$ for 6 hours. In Saskatchewan, $88.8 \%, 92.6 \%$ and $92.9 \%$ of the population lived within 3.5 , 4.5 and 6 hours, respectively, from a stroke centre via selfdriving. These data reflect the larger proportion of the populations of these provinces living in rural and remote areas, where EMS and stroke-enabled hospital access is geographically challenging (Figure 3 ).

Assuming a 5-hour travel time via EMS from base to patient and then from patient to hospital, $77.0 \%$ of the country's population had access to a site that participated in the ESCAPE trial, $79.8 \%$ had access to a proposed additional endovascular site, and $94.0 \%$ had access to a current or proposed endovascular site (Table 3).

\section{Interpretation}

A high proportion of the population of the 8 provinces studied has potential access to acute stroke treatment with intravenously administered alteplase, and $84.7 \%-99.8 \%$ have access to acute endovascular therapy in the provinces where this is available. However, we found substantial variability. With private transport, $86.4 \%-100.0 \%$ of the population lives within a 4.5-hour drive of a stroke centre. With EMS (to the patient's home and then to hospital), 47.1\%-96.4\% live within 4.5 hours, and $53.3 \%-96.8 \%$ live within 6 hours. These findings suggest that most but not all Canadians have access to hyperacute stroke services.

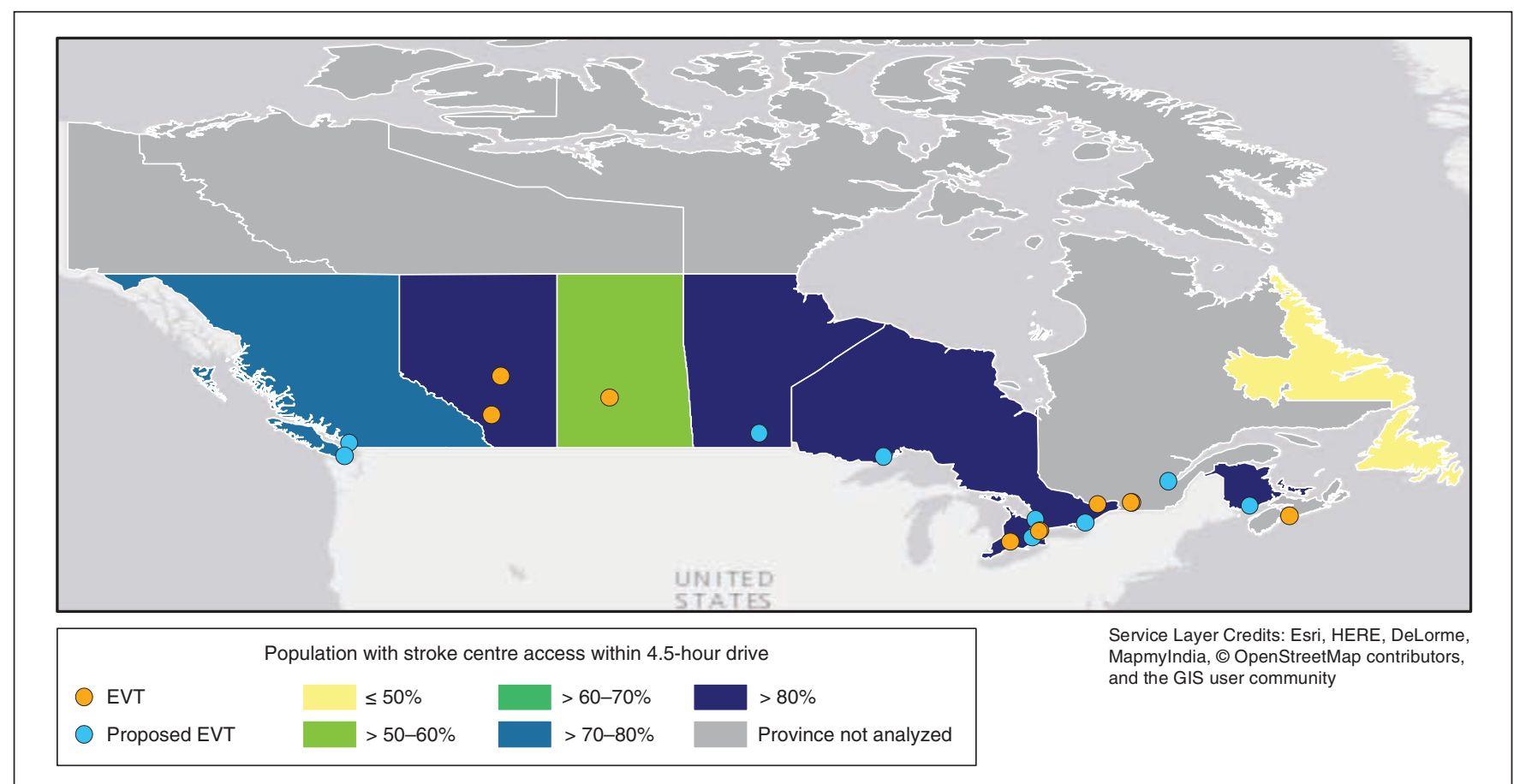

Figure 3: Map of Canada, showing the current comprehensive stroke centres capable of providing endovascular thrombectomy (EVT). Colour-coding by province indicates the proportion of the population that has access to a stroke centre within 4.5 hours via emergency medical services road transport. 
Table 3: Access to acute endovascular treatment services in Canada

\begin{tabular}{|c|c|c|c|c|}
\hline \multirow[b]{2}{*}{ Province* $^{*}$} & \multirow[b]{2}{*}{$\begin{array}{l}2011 \text { census } \\
\text { population }\end{array}$} & \multicolumn{3}{|c|}{ No. (\%) of people within 5-hour drive via emergency medical services } \\
\hline & & $\begin{array}{l}\text { Initial ESCAPE }{ }^{14} \\
\text { endovascular } \\
\text { thrombectomy sites } \\
\quad(n=11)\end{array}$ & $\begin{array}{l}\text { New/proposed } \\
\text { endovascular } \\
\text { thrombectomy sites } \\
\qquad(n=10)\end{array}$ & $\begin{array}{c}\text { All endovascular } \\
\text { thrombectomy sites } \\
\qquad(n=21)\end{array}$ \\
\hline British Columbia & 4400052 & $53927(1.2)$ & $3677489(83.6)$ & $3728112(84.7)$ \\
\hline Alberta & 3645081 & $3410423(93.6)$ & - & $3410423(93.6)$ \\
\hline Saskatchewan & 1033489 & $932229(90.2)$ & $32553(3.1)$ & $932229(90.2)$ \\
\hline Manitoba & 1208268 & $12557(1.0)$ & $1122016(92.9)$ & $1122099(92.9)$ \\
\hline Ontario & 12851791 & $12315533(95.8)$ & $12545652(97.6)$ & $12545652(97.6)$ \\
\hline Quebec & 7902991 & $7025574(88.9)$ & 7336516 (92.8) & 7403935 (93.7) \\
\hline New Brunswick & 751161 & 535704 (71.3) & $740672(98.6)$ & $740672(98.6)$ \\
\hline Nova Scotia & 921727 & $871989(94.6)$ & 620015 (67.3) & $871989(94.6)$ \\
\hline Prince Edward Island & 140204 & 139965 (99.8) & $139965(99.8)$ & $139995(99.8)$ \\
\hline Canada & 32854764 & 25297901 (77.0) & 26214878 (79.8) & 30895106 (94.0) \\
\hline
\end{tabular}

In Canada in 2011, 69.0\% of patients with stroke admitted to hospital were transported by EMS. The Canadian Stroke Best Practice Recommendations have established a target for EMS transport of $80 \%$ of patients. ${ }^{5}$ Findings from the North Carolina Stroke Care Collaborative showed that arrival by EMS (v. private transport) was associated with faster access to brain imaging and faster interpretation of these images. ${ }^{15}$ Canada's vast geography, challenging landscapes and climate make it almost impossible to get all patients to hospital in time for treatment, even with fast door-to-treatment times. People living in rural and remote locations in general have challenges accessing EMS for urgent conditions. Patterns of access limitation similar to those observed in the current study have been seen in care for acute coronary syndromes and trauma care. ${ }^{16}$

The ESCAPE trial delivered rapid endovascular treatment to patients who had had a stroke and had proximal intracranial occlusion on imaging at 11 sites in Canada, improving clinical outcomes and reducing mortality. ${ }^{14}$ The key difference between the trial sites and the current analysis is that the proposed sites cover the large population centres of Vancouver and Winnipeg. One pan-Canadian study similarly used geospatial tools to estimate the proportion of the population with $60-, 90-$ and 120 -minute access to a hospital with the minimum requirements to treat stroke (a third- or fourth-generation computed tomography scanner, a neurologist and an emergency physician on staff). ${ }^{17}$ Based on 1991 and 1996 census counts, $67.3 \%$ of Canadians lived within 60 minutes of such a centre, $78.2 \%$ within 90 minutes and $85.3 \%$ within 120 minutes. An Ontario study showed that access to hospitals with specialized services (including interventional cardiology centres) varied by geography, with rural areas having significantly less access..$^{18}$ Overall, $40.5 \%$ of the population had access to a hospital with specialized services within 30 minutes, $72.2 \%$ had access within
1 hour, and $96.5 \%$ had access within 4 hours. van Rheenen ${ }^{19}$ used geospatial tools to estimate the change in the proportion of the Alberta population with access to a stroke centre after the province increased the number of such centres (between 2002 and 2010). The proportion of Albertans with geographic access within 15 minutes by ground transport increased from $47.5 \%$ to $53.8 \%$, and the proportion with access within 75 minutes increased from $84.6 \%$ to $93.9 \%$.

The geospatial approach can be useful to inform capacity planning. For example, extending transport time windows from 4 to 6 hours makes a bigger difference in increasing access to acute stroke treatment in Newfoundland and Labrador than in Saskatchewan. By plotting potential sites, decision-makers can objectively determine which locations would have greater population reach. This information, in turn, would need to be combined with hospital capability to care for patients with stroke following acute treatment, resources (human and equipment), expertise and expected annual volumes to justify location selection.

\section{Limitations}

The use of geospatial tools to estimate access to stroke services is a relatively new application. These analyses depend on accurate road data and speed of travel by ambulances. One study in Calgary showed that the actual time to the stroke centre, as recorded by EMS, was consistently higher than predicted by the geospatial tools, usually by $10-20$ minutes. ${ }^{20}$ Again, the error was not randomly distributed, with areas outside the city having the highest underestimates. By adjusting model assumptions to correct for this underestimate, Patel ${ }^{20}$ estimated that access within 60 minutes to invasive cardiac services was available for $56.3 \%$ of the Canadian population, with a low of $13.5 \%$ in New Brunswick and a high of $62.7 \%$ in Ontario. A 
similar study from Alberta showed that travel time predicted with geospatial tools was about $70 \%$ of actual EMS time. The greatest discrepancies occurred during the winter months and peak morning and afternoon rush hour, reflecting real-time conditions (poor weather, traffic) not often accounted for in a geographic information system. ${ }^{19}$ The simple assumption that the population is equally distributed around a forward sortation area will bias the results in rural areas. Instead, it is expected that a population will be heavily distributed around the road network. This bias is greater in lower-population areas, thus limiting the error. We assume that an ambulance is dispatched immediately after a stroke occurs and travels at an average road speed, and that EMS spend no time at the patient's residence. This will lead to errors associated with ambulance speed compared to the average road speed (expected to be higher), with estimating traffic (the true travel time might be longer or shorter than the road averages applied, given time of day and traffic disruptions) and with ignoring delays at the patient's location (notably, delays associated with travel within large buildings). Some provinces and hospitals have access to air ambulance, and we did not consider this possibility in our analysis. Our estimates also assume that patients or witnesses will contact EMS as soon as stroke symptoms begin. Canadian monitoring data reveal significant delays in first contact with EMS or first presentation to an emergency department by private transport for patients who have experienced a stroke. ${ }^{1}$ These delays may result in reduced numbers of patients arriving in specified time windows, even if they reside within a 6-hour drive of a stoke centre. Finally, estimates for provinces with a greater rural population may be underestimated because local private EMS providers could not be identified.

\section{Conclusion}

Our study reports on the predicted access of the Canadian population to stroke centres that provide time-sensitive acute treatments. Most Canadians live within 6 hours by road to a stroke centre. Coordinated systems of care and ambulance bypass agreements must continue to evolve to ensure maximal access to hyperacute stroke services. The public needs to be aware of the signs of stroke and contact EMS without delay to maximize eligibility for these time-sensitive treatments. Geospatial analysis may help estimate coverage for specific stroke services (e.g., intraarterial clot retrieval) and other medical or surgical emergencies. This method could be broadly applied in health care to inform decisions about resource allocation to maximize population access and to identify gaps in service accessibility across a range of health conditions and across geographic regions.

\section{References}

1. Access to stroke care: the critical first hours. The Heart and Stroke Foundation 2015 Stroke Report. Ottawa: Heart and Stroke Foundation; 2015. Available: www.strokebestpractices.ca/wp-content/uploads/2015/06/HSF-2015-Stroke -Report_EN-FINAL1.pdf (accessed 2017 Apr. 10)

2. The National Institute of Neurological Disorders and Stroke rt-PA Stroke Study Group. Tissue plasminogen activator for acute ischemic stroke. $N$ Engl 7 Med 1995;333:1581-7.

3. Saver JL, Levine SR. Alteplase for ischaemic stroke - much sooner is much better. Lancet 2010;375:1667-8.

4. Hacke W, Kaste M, Bluhmki E, et al. Thrombolysis with alteplase 3 to 4.5 hours after acute ischemic stroke. N Engl 7 Med 2008;359:1317-29.
5. Casaubon LK, Boulanger JM, Blacquiere D, et al. Canadian Stroke Best Practice Recommendations: Hyperacute Stroke Care guidelines, update 2015. Int 7 Stroke 2015;10:924-40.

6. Prabhakaran S, McNulty M, O'Neill K, et al. Intravenous thrombolysis for stroke increases over time at primary stroke centers. Stroke 2012;43:875-7.

7. Prabhakaran S, Ward E, John S, et al. Transfer delay is a major factor limiting the use of intra-arterial treatment in acute ischemic stroke. Stroke 2011;42: 1626-30.

8. Evenson KR, Foraker RE, Morris DL, et al. A comprehensive review of prehospital and in-hospital delay times in acute stroke care. Int 7 Stroke 2009;4:187-99.

9. Saver JL. Time is brain - quantified. Stroke 2006;37:263-6.

10. Saver JL, Goyal M, van der Lugt A, et al. Time to treatment with endovascular thrombectomy and outcomes from ischemic stroke: a meta-analysis. $7 A M A$ 2016;316:1279-88.

11. Wieczorek WF, Delmerico AM. Geographic information systems. Comput Stat 2009; 1:167-86.

12. Longley PAGM, Maguire DJ, Rhind DW. Geographic information systems and science. West Sussex (UK): John Wiley \& Sons; 2005.

13. Boulos MN. Towards evidence-based, GIS-driven national spatial health information infrastructure and surveillance services in the United Kingdom. Int 7 Health Geogr 2004;3:1.

14. Goyal M, Demchuk AM, Menon BK, et al. Randomized assessment of rapid endovascular treatment of ischemic stroke. N Engl 7 Med 2015;372:1019-30.

15. Patel MD, Rose KM, O’Brien EC, et al. Prehospital notification by emergency medical services reduces delays in stroke evaluation: findings from the North Carolina Stroke Care Collaborative. Stroke 2011;42:2263-8.

16. Lawson FL, Schuurman N, Oliver L, et al. Evaluating potential spatial acces to trauma center care by severely injured patients. Health Place 2013;19:131-7.

17. Scott PA, Temovsky CJ, Lawrence K, et al. Analysis of Canadian population with potential geographic access to intravenous thrombolysis for acute ischemic stroke. Stroke 1998;29:2304-10.

18. Glazier RH, Gozdyra P, Yeritsyan N. Geographic access to primary care and hospital services for rural and northern communities: report to the Ontario Ministry of Health and Long-Term Care. Toronto: Institute for Clinical Evaluative Sciences; 2011.

19. van Rheenen SM. A spatial epidemiological analysis of stroke in Alberta, Canada, using GIS [dissertation]. Calgary: University of Calgary; 2015.

20. Patel AB. Evaluating geographic access to cardiac catheterization facilities to inform regionalized models of care for acute myocardial infarction [dissertation]. Calgary: University of Calgary; 2012.

Affiliations: Calgary Stroke Program (Eswaradass, Hill), Department of Clinical Neurosciences, Cumming School of Medicine; Departments of Radiology (Hill), Community Health Sciences (Hill) and Medicine (Hill), Cumming School of Medicine, University of Calgary; Hotchkiss Brain Institute (Hill), Calgary, Alta.; Heart and Stroke Foundation (Lindsay), Ottawa, Ont.; Neurology Division (Swartz), Department of Medicine, Sunnybrook Health Sciences Centre, University of Toronto, Toronto, Ont.; Geosyntec Consultants (Rosen), Guelph, Ont.

Contributors: Prasanna Eswaradass was lead author for the manuscript including drafting and revision. Patrice Lindsay, Richard Swartz and Michael Hill conceptualized the study, developed the analysis plan and contributed to writing and revising the manuscript. Jamey Rosen developed the geospatial methodology and conducted all geospatial analysis, interpreted the data and contributed to writing the manuscript. All of the authors approved the final version to be published and agreed to act as guarantors of the work.

Competing interests: Michael Hill has received grant support from Alberta Innovates - Health Solutions, the Canadian Institutes of Health Research, the Heart and Stroke Foundation of Canada and the US National Institute of Neurological Disorders and Stroke. He reports personal fees from Merck, nonfinancial support from HoffmannLa Roche Canada, and grants from Covidien (Medtronic), Boehringer Ingleheim, Medtronic and NoNO outside the submitted work. He has a patent pending (no. 62/086,077) for Systems and Methods for Assisting in Decision-Making and Triaging for Acute Stroke Patients, owns stock in Calgary Scientific Incorporated, a company that focuses on medical imaging software, and is a director of the Canadian Neurological Sciences Federation, a not-for-profit group. Richard Swartz reports a Heart and Stroke Foundation of Canada New Investigator Award during the conduct of the study. No other competing interests were declared.

Supplemental information: For reviewer comments and the original submission of this manuscript, please see www.cmajopen.ca/content/5/2/ E454/suppl/DC1 